\title{
Effect of Matrix Content on Mechanical and Thermal Properties of High Graphene Content Composites
}

\author{
F. Cilento ${ }^{1}$, A. Martone ${ }^{2 a}$, F. Cristiano ${ }^{3}$, A. Fina ${ }^{4}$, M. Giordano ${ }^{2}$ \\ ${ }^{1}$ Department of Chemical, Materials and Production Engineering, University of Naples Federico II, Napoli, Italy \\ ${ }^{2} I P C B$, Institute of Polymers, Composite and Biomaterials, CNR, Portici, Italy \\ ${ }^{3} \mathrm{Nanesa}$ srl, Arezzo, Italy \\ ${ }^{4}$ Department of Applied Science and Technology, Politecnico di Torino, Alessandria, Italy
}

\begin{abstract}
Recently, biomimetic brick and mortar composites (B/M) are gathering great attention due to their outstanding properties. The use of graphene as bricks is expected to achieve good mechanical performances combined with remarkable thermal diffusivity making them optimal candidates for heat spread applications. Macroscopic composites ( $1 \mathrm{~mm}$ thick) have been manufactured at different filler content (up to $100 \%$ vol\%) and their morphology have been investigated by scanning electron microscopy. Bending test have been carried out on samples for measuring the effect of the polymer amount on the composite. The thermal diffusivity has been investigated, both in plane and cross plane, by light flash analysis (LFA). Coupons showed a well aligned inner structure at each resin content, however the effective performances depends on the capability of stress transfer.
\end{abstract}

\section{Introduction}

Freestanding paper-like materials base on nanoscale lamellar fillers have attracted extensive interest thank to their intrinsic properties. In fact, such class of materials borrows specific properties from the two dimensional (2D) nano-filler together with special features related to the material architecture[1]-[3]. Due to its multifunctional properties, graphene based twodimensional sheets are ideal candidate for developing protective layers, chemical filters, adhesive layers, flexible sensing and energy devices, and wearable electronics[4], [5].

The challenge is then to retain at the macroscale the mechanical and thermal properties of the filler. In the case of lamellar fillers paper-like structures allow to reproduce on the macroscopic scale the behaviour of the nanoscale reinforcement, in addition, based on the manufacturing procedure additional features such as foldability, permeability, barrier properties should be engraved to the material[6], [7]. Both graphene oxide [8]-[10] and reduced graphene oxide [9], [11], [12] have assembled by means of layer-by-layer technology to form a very thin nanolaminate film (few tens microns thickness) showing exceptionally rich multifunctional properties [13]. The inner architecture of nacre-like composites is quite different respect to the conventional paper-like composites. Main differences between the two architecture are the porosity, i.e. bucky papers possess connected micro porosity, and a lower degree of in-plane oriented nanoplatelets [14]. Li et al. [15] and Biswas and
Drzal [16] both fabricated Langmuir-Blodgett thin films from exfoliated graphite. However, the LangmuirBlodgett approach is not suitable for a thickness in the micron range and the porosity of the film is usually hard to control, making it not an adequate solution for composites applications. Wu and Dzral [8] fabricated a self-standing graphite paper consisting of graphite nanoplatelets (GNPs) with controlled porosity. Both thermoset and thermoplastic matrices were used to impregnate the porous GNP neat paper and an interesting tensile modulus $(22 \mathrm{GPa})$ was attained by the nanolaminate at a minimum content of $20 \%$ of the polymer matrix. Recently, Li et al [17] produced high content GNP polyetherimide, PEI, matrix paper by filtration and hot-press. The GNP paper showed an improvement of the damping factor in respect to the neat PEI and the pure GNP.

In this work, prepregs made at high content of GNP have been fabricated at different GNP content from $60 \%$ vol up to $100 \%$ vol. Morphological analysis showed that films have a nacre like hierarchical structure. Thermal diffusivity was investigated both in-plane and cross-plane showing outstanding performances. We further investigate the effect of the matrix content on thermal diffusivity of films and flexural elastic modulus of the nanolaminates. The results revealed that these class of composites are promising candidates for heat spread applications since they combine good mechanical performances and remarkable thermal diffusivity.

\footnotetext{
${ }^{\mathrm{a}}$ Corresponding author: alfonso.martone@cnr.it
} 


\section{Materials and methods}

\subsection{Materials}

Graphite nanoplatelets, namely G2NAN, were kindly supplied by Nanesa srl. The single particle has a lateral size and thickness of $30 \mu \mathrm{m}$ and $14 \mathrm{~nm}$ respectively and a specific surface area $(B E T)>30 \mathrm{~m}^{2} / \mathrm{g}$.

HexFlow ${ }^{\circledR}$ RTM6 is a degassed monocomponent resin purchased from Hexcel, specially developed to fulfil the requirements of the aerospace and space industry in advanced resin transfer moulding processes (RTM). It has been characterized by differential scanning calorimeter (DSC) and thermal gravimetric analysis (TGA). It exhibits high glass transition temperatures, around $190^{\circ} \mathrm{C}$, and a reticulation peak at $241^{\circ} \mathrm{C}$ with an energy of 470 $\mathrm{J} / \mathrm{g}$. Thus, a cure cycle at $160^{\circ} \mathrm{C}$ for $90 \mathrm{~min}$ is chosen, followed by a post-cure cycle at $180^{\circ} \mathrm{C}$ for 1 hour.

\subsection{Experimental methods}

The actual polymeric matrix content has been investigated through TGA Q500. The analysis were performed in inert atmosphere, using nitrogen gas, with a temperature ramp of $10^{\circ} \mathrm{C} / \mathrm{min}$ from room temperature to $800^{\circ} \mathrm{C}$. The weight loss is evaluated at $600^{\circ} \mathrm{C}$, temperature at which the percent residue from heating the pure resin is $10.3 \%$.

The film thermal diffusivity has been investigated through laser flash analysis (LFA), with NETZSCH instrument.

Scanning electron microscopy (SEM) FEI Quanta 200 FEG has been employed to observe the cross section of the laminates, in order to study the orientation of the particles and the adhesion between the plies. Samples were fractured in nitrogen in order to have a picture of the laminates section.

Bulk laminates have been manufactured by Compression moulding by platen press (P200E Collin): different GNP pre-impregnated films are stacked, positioned between two metallic caul plates and confined by a frame. The laminate is cured for $90 \mathrm{~min}$ at $160^{\circ} \mathrm{C}$ with a pressure of 40 bar.

Mechanical characterization of bulk GNP composites was performed by a DMA-Q800 equipped with 3 point bending clamp with a displacement rate of $500 \mu \mathrm{m} / \mathrm{min}$. Data were elaborated according to the ASTM D790 standard for flexural behaviour of composites.

Table 1. Actual composition in GNP/epoxy films.

\begin{tabular}{|c|c|c|c|c|c|}
\hline & $\begin{array}{c}\mathbf{6 0} \\
\text { wt. \%G } \\
\text { NP }\end{array}$ & $\begin{array}{c}\mathbf{7 0} \\
\text { wt. \% } \\
\text { GNP }\end{array}$ & $\begin{array}{c}\mathbf{8 0} \\
\text { wt. \% } \\
\text { GNP }\end{array}$ & $\begin{array}{c}\text { 90 } \\
\text { wt. \% } \\
\text { GNP }\end{array}$ & $\begin{array}{c}\mathbf{1 0 0} \\
\text { wt. \% } \\
\text { GNP }\end{array}$ \\
\hline Actual (wt. \%) & 61 & 69 & 78 & 92 & 100 \\
\hline Density (g/cm & 1.4 & 1.5 & 1.4 & 1.4 & 1.8 \\
\hline Void (\%) & 7 & 6 & 18 & 24 & 10 \\
\hline GNP (vol \%) & 42 & 53 & 67 & 82 & 100 \\
\hline
\end{tabular}

\section{Results and discussion}

\subsection{Thermal properties}

The GNP platelet have anisotropic properties in-plane direction respect to the cross plane, the alignment of GNP particles can help GNP films to achieve maximum performance at the in-plane direction. It is expected that GNP films exhibits elastic modulus, electrical and thermal conductivity reproducing the anisotropy of the GNP particles. The best the alignment achieved will led to improved performances up the bare GNP particle.

Generally, the three kinds of properties are positively related to the density of the sample, Table 1 shows the achieved density for GNP films at different polymeric binder content.

Thermal gravimetrical analysis was carried out to estimate the actual GNP content within GNP films. To eliminate the effect of porosity and obtain best properties, the GNP films were also mechanically compressed during consolidation.

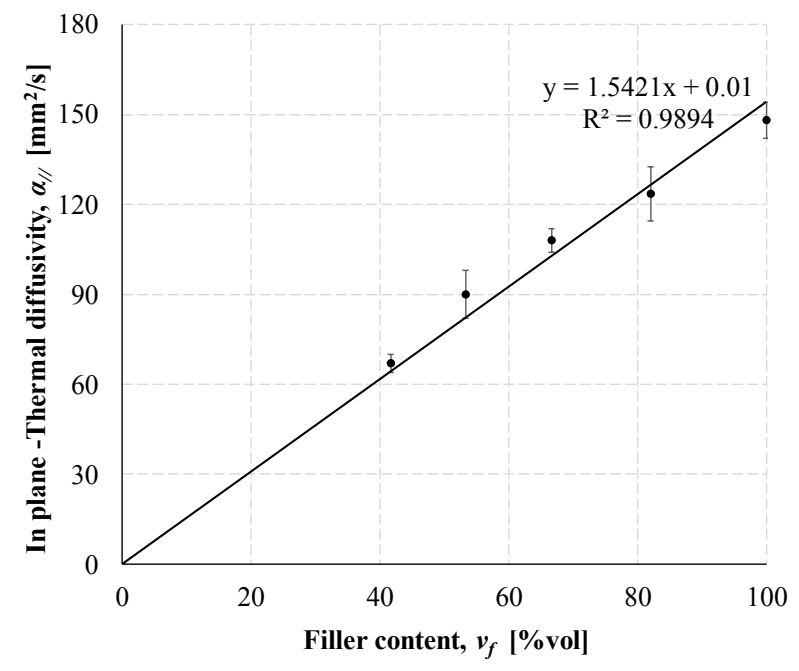

a)

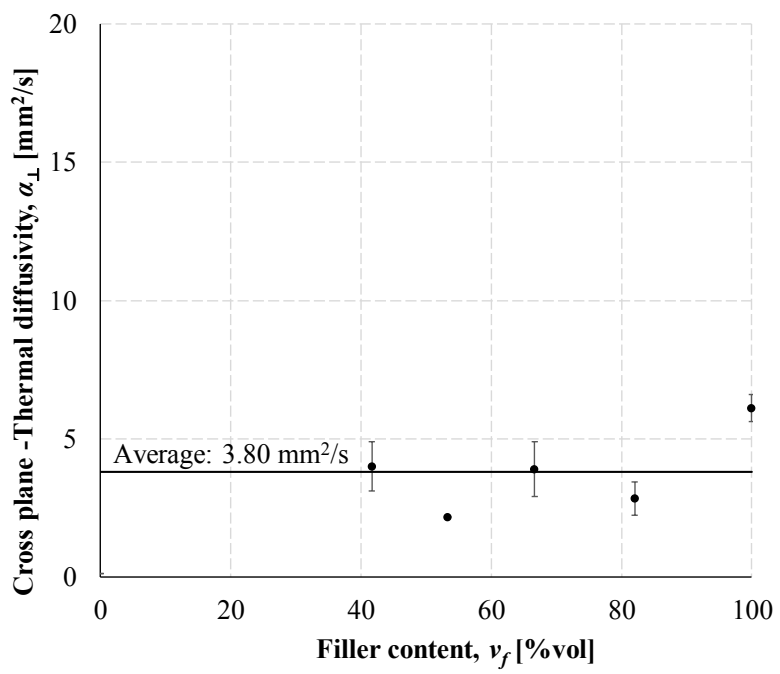

b)

Figure 1. Thermal diffusivity trend for different filler content: a) In plane; b) Cross plane. 
The in plane thermal diffusivity of GNP/Epoxy papers linearly increase with the increasing filler content (Figure 1a) as it is expected. In fact, the addiction of polymer, reduces the thermal diffusivity, it means that for high filler content, there are many GNP-GNP bridges, which allow the heat transfer. Whereas the cross plane thermal diffusivity (Figure 1b) is quite constant and slightly increase in the case of pure GNP paper, indicating that the polymer does not affect the thermal properties in the cross plane direction.

The higher is the filler content the higher is the in plane thermal diffusivity (Figure 1a), while cross-plane diffusivity has not a real trend fluctuating close to the thermal conductivity of the graphite [18].

The adopted fabrication route was able to reproduce the anisotropic thermal properties of the graphite nanoplatelet, the only issue is the high porosity detected. The voids negatively affects the cross-plane diffusivity, while the good alignment guaranteed by the calendering stage led to good correlation between the resin content and the material diffusivity.

\subsection{Mechanical performances of films and layered GNP rich composites}

Residual porosity negatively affects the mechanical properties of composites, the compaction pressure has not been uniformly distributed during the consolidation stage. In order to improve the alignment of GNP within the material and to decrease the void content, during the processing of thick GNP composites layer are stacked up to a final thickness $30 \%$ higher than the desired final thickness and the compression moulded at pressure of 40 bar. A set of coupons were manufactured at a nominal thickness of $1 \mathrm{~mm}$ in order to investigate the effect of resin content on the final mechanical performances.

Figure 2 shows fracture surface at a matrix content of $\sim 30 \mathrm{vol} \%$. Here, the alignment of GNP in the plane is greatly improved, the cross section exhibits a uniform texture. Even if the overall distribution of lamellar particle is good some voids are still present. Empty areas should weak points affecting the bonding between layers. Mechanical tests carried out on laminates reproduced a similar behaviour as observe for film, even if the higher compaction load lead to improved final mechanical performances. The increase stiffness probably is related to the higher ordering achieved and to the decrease of void content.

Figure 3 shows the results of flexural tests conducted on laminates. The picture reports the evolution of the elastic modulus at different filler content. At GNP loading lower than $70 \mathrm{vol} \%$ the elastic modulus follows the direct rule of mixture, while at higher filler content (>70 vol\%) the elastic modulus abruptly drops, then starts to increase with a different slope. This jagged-like behaviour of the young modulus can be associated either to the achieving of a critical stress corresponding to the exfoliation of the graphitic layers either to a too thin interphase, due to low resin content, which is not able to efficiently transfer load.

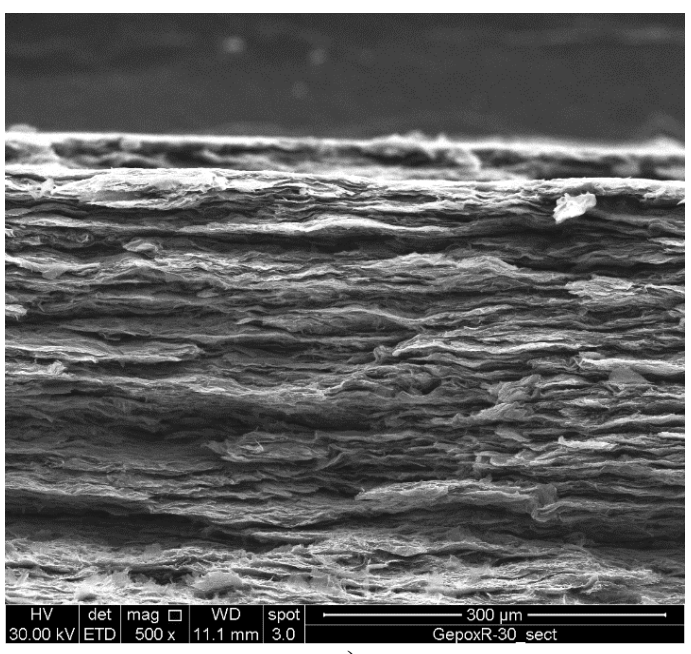

a)

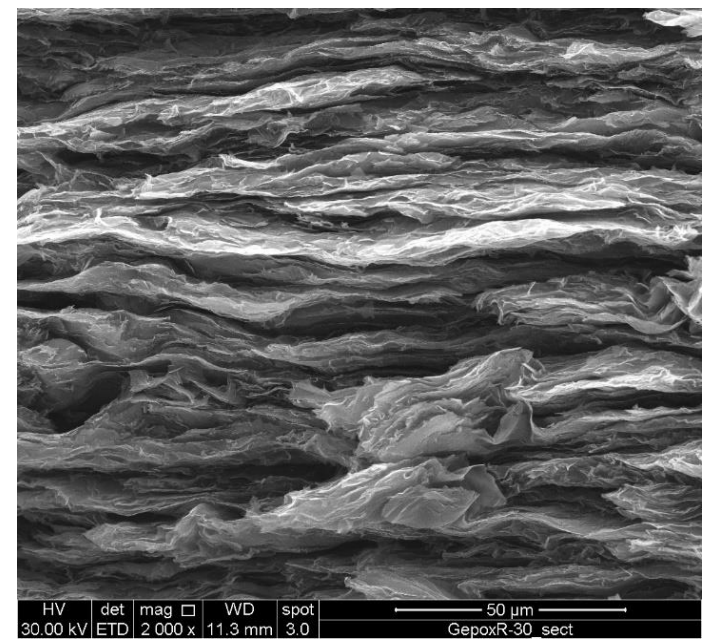

b)

Figure 2. SEM Fracture surfaces, actual composition GNP/Epoxy 66/33vol\%.

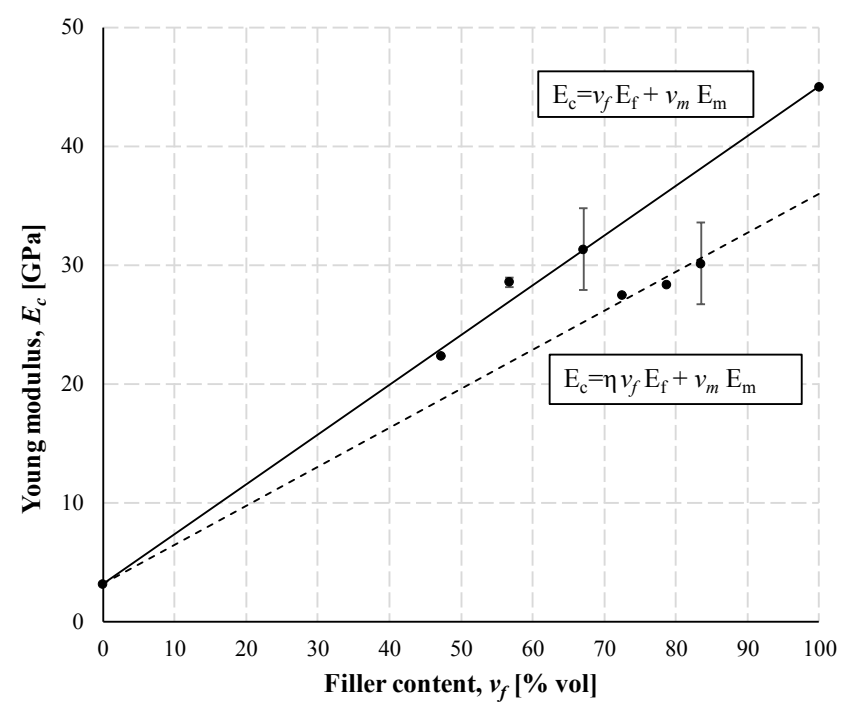

Figure 3. Flexural elastic modulus of GNP/Epoxy laminates at different filler content.

The analysis of modulus vs GNP content data based on simplified shear lag model [19] reveals that in the 
range $40-70 \mathrm{vol} \%$ the reinforcement effect is maximum, while in the range between $70-90$ vol $\%$ drops by $20 \%$. The latter scenario is reproduced both for films and both for thick laminates. Data in Figure 3 are divided in two separate set, fitted respectively by models with a relative efficiency of 1 and 0.8 .

\section{Conclusions}

In the present paper a procedure for manufacturing GNP rich films have been described. The proposed methods allows to wet GNP by a polymeric binder (i.e. epoxy, PU). Films made by epoxy and GNP are suitable for further manufacturing of thick composites made by compression moulding.

Main advantage of such class of composite is to reproduce on meso and macroscale the properties of lamellar nanofiller used as building block. Thermal diffusivity measurement reported an outstanding thermal conductivity on the particle plane while cross-plane measurement shows a global conductivity similar to the GNP brick independent from the resin phase.

Mechanical tests, conducted on both GNP films and $1 \mathrm{~mm}$ thick laminates (with more than 50 vol\%) showed a jagged increase of composite elastic modulus. Based on simplified shear lag model two different trend were highlighted on data in the range $50-70 \mathrm{vol} \%$ and in the range 70-90 vol\%, where a drop in reinforcement efficiency drop of $20 \%$ has been experienced.

\section{Acknowledgments}

Activities were carried out in the framework of an Italian grant "FIRE - graphene based fire protection coatings". $\mathrm{PhD}$ grant of Fabrizia Cilento is supported by the programme "PON Ricerca e Innovazione 2014-2020"

\section{References}

1 Y. Yao and J. Ping, "Recent advances in graphene-based freestanding paper-like materials for sensing applications," TrAC - Trends in Analytical Chemistry, vol. 105. Elsevier, pp. 7588, (2018).

2 D. F. Schmidt, "Nanolaminates - Bioinspired and beyond," Mater. Lett., vol. 108, pp. 328-335, (2013).

3 M. R. Ricciardi, A. Martone, F. Cristiano, F. Bertocchi, and M. Giordano, "Nacre-like composites made by graphite nanoplatelets," in AIP Conference Proceedings, vol. 1981, no. 1, p. 020072, (2018).

4 S. Wan, J. Peng, L. Jiang, and Q. Cheng, "Bioinspired Graphene-Based Nanocomposites and Their Application in Flexible Energy Devices," Adv. Mater., vol. 28, no. 36, pp. 78627898, (2016).

$5 \quad$ Y. Zhang et al., "Graphene-based artificial nacre nanocomposites," Chem. Soc. Rev., vol. 45, no. 9, pp. 2378-2395, (2016).
Structured Graphene Paper for High Performance Electrode Materials," Adv. Mater., vol. 24, no. 8, pp. 1089-1094, Feb. (2012).

Z. Tang, N. A. Kotov, S. Magonov, and B. Ozturk, "Nanostructured artificial nacre," Nat. Mater., vol. 2, no. 6, pp. 413-418, (2003).

H. Wu and L. T. Drzal, "Graphene nanoplatelet paper as a light-weight composite with excellent electrical and thermal conductivity and good gas barrier properties," Carbon N. Y., vol. 50, no. 3, pp. 1135-1145, (2012).

9 P. Kumar, F. Shahzad, S. Yu, S. M. Hong, Y. H. Kim, and C. M. Koo, "Large-area reduced graphene oxide thin film with excellent thermal conductivity and electromagnetic interference shielding effectiveness," Carbon N. Y., vol. 94, pp. 494-500, (2015).

10 B. Shen, W. Zhai, and W. Zheng, "Ultrathin flexible graphene film: An excellent thermal conducting material with efficient EMI shielding," Adv. Funct. Mater., vol. 24, no. 28, pp. 4542-4548, (2014).

11 A. Yu, P. Ramesh, X. Sun, E. Bekyarova, M. E. Itkis, and R. C. Haddon, "Enhanced thermal conductivity in a hybrid graphite nanoplatelet Carbon nanotube filler for epoxy composites," $A d v$. Mater., vol. 20, no. 24, pp. 4740-4744, (2008).

12 N. J. Song, C. M. Chen, C. Lu, Z. Liu, Q. Q. Kong, and R. Cai, "Thermally reduced graphene oxide films as flexible lateral heat spreaders," $J$. Mater. Chem. A, vol. 2, no. 39, pp. 16563-16568, (2014).

13 Y. Liu, B. Xie, Z. Zhang, Q. Zheng, and Z. Xu, "Mechanical properties of graphene papers," $J$. Mech. Phys. Solids, vol. 60, no. 4, pp. 591-605, (2012)

14 Z. U. Khan, A. Kausar, H. Ullah, A. Badshah, and W. U. Khan, "A review of graphene oxide, graphene buckypaper, and polymer/graphene composites: Properties and fabrication techniques," J. Plast. Film Sheeting, vol. 32, no. 4, pp. 336-379, (2016).

15 X. Li et al., "Highly Conducting Graphene Sheets and Langmuir-Blodgett Films."

16 S. Biswas and L. T. Drzal, "A novel approach to create a highly ordered monolayer film of graphene nanosheets at the liquid-liquid interface," Nano Lett., vol. 9, no. 1, pp. 167-172, (2009).

17 X. Li et al., "Preparation and Characterization of High Content Graphene NanoplateletPolyetherimide Paper," J. Nanosci. Nanoeng., vol. 1, no. 4, pp. 252-258, (2015).

18 H. O. Pierson and H. O. Pierson, "3 - Graphite Structure and Properties," in Handbook of Carbon, Graphite, Diamonds and Fullerenes, pp. 43-69 (1993).

19 R. J. Young et al., "The mechanics of reinforcement of polymers by graphene nanoplatelets," Compos. Sci. Technol., vol. 154, pp. 110-116, (2018). 